\title{
Cartilage macromolecules in knee joint synovial fluid. Markers of the disease course in patients with acute oligoarthritis
}

\author{
Elisabet Lindqvist, Tore Saxne
}

\begin{abstract}
Objective-To investigate the development of chronic joint symptoms in patients presenting with acute oligoarthritis including knee joint synovitis with effusion and explore whether prognostic information can be derived from initial synovial fluid concentrations of aggrecan and cartilage oligomeric matrix protein (COMP) for development of chronic joint symptoms.

Methods-Retrospective follow up of 25 patients identified in a bank of knee joint synovial fluids collected consecutively from patients presenting with knee joint synovitis and symptoms from at most three additional joints and in whom no diagnosis could be established at presentation.

Results-The 10 patients who developed chronic joint symptoms were characterised by lower knee joint synovial fluid concentrations of aggrecan as well as lower aggrecan/COMP ratios $(p<0.001)$ than the 15 patients who had a transient arthritis. No other clinical or laboratory differences between the groups were apparent at the time of presentation.

Conclusions-The synovial fluid content of aggrecan is a potential tool in acute arthritis for distinguishing patients with a benign disease course from those who will develop a chronic joint disorder.
\end{abstract}

(Ann Rheum Dis 1997;56:751-753)

Arthritis with acute onset frequently presents a diagnostic challenge in rheumatology. ${ }^{1}$ After identifying the rare cases of septic arthritis and the cases of crystal induced arthritis, most of the remaining patients will initially be diagnosed as having undifferentiated noninfectious inflammatory arthritis. In some of these patients the disease course will reveal the diagnosis, whereas in others the diagnosis will remain unknown. In some patients the acute arthritis will resolve completely while others will develop a chronic joint disorder for example, rheumatoid arthritis (RA). Conventional clinical or laboratory variables often fail to reveal the diagnosis in the acute phase, or predict the subsequent course of the arthritis. ${ }^{2}$ Thus, it is not possible to give the patient reliable prognostic information and importantly, it is impossible to initiate aggressive treatment in cases prone to rapid functional or structural deterioration resulting from a chronic joint disease. As it is increasingly believed that such early treatment is beneficial the lack of diagnostic and prognostic instruments applicable in the acute stage may preclude potentially preventive treatment. ${ }^{3}$

Synovial fluid analyses of joint matrix macromolecules represent a novel tool for the study of tissue involvement in arthritis. ${ }^{4}$ Promising results regarding the diagnostic and prognostic utility of such measurements have been obtained. ${ }^{5}$ Notably, in acute stages of reactive arthritis and crystal induced arthropathies with normal radiographic appearance of the knee joints, high concentrations of aggrecan, the large aggregating cartilage proteoglycan, have been found in synovial fluid. ${ }^{6}$ The concentrations in these conditions significantly exceeded the values found in RA patients with normal knee joint radiographs and a duration of synovitis comparable with that in reactive arthritis. ${ }^{6}$ Subsequently, as the joint symptoms subsided in the patients with reactive arthritis, decreasing synovial fluid concentrations of aggrecan were found. ${ }^{7}$ In RA, the synovial fluid aggrecan concentrations are highest in patients with radiographically well preserved joints and lower values are found in stages with pronounced joint damage. ${ }^{6}$ In contrast, the synovial fluid content of another primarily cartilage derived macromolecule, cartilage oligomeric matrix protein (COMP), does not appear to vary considerably in different phases of reactive arthritis or RA. ${ }^{78}$ The ratio between the concentrations of these markers in synovial fluid has been used to reduce the confounding effect of variations in amounts of retrievable fluid, regardless of the fact that the clearance rates of the molecules may vary. ${ }^{8}$

In this retrospective study we have investigated the development of chronic joint symptoms in patients initially presenting with acute oligoarthritis including a synovitis of the knee joint requiring joint fluid aspiration and where a diagnosis could not be established at presentation using clinical or routinely used laboratory variables. Furthermore, we explored the concentration in synovial fluid of aggrecan and COMP, as possible predictors of persisting arthritis.

\section{Methods}

PATIENTS

A computer search of a bank of synovial fluid samples collected consecutively from patients in whom arthrocentesis had been performed 
Table 1 Characteristics of patients with transient arthritis $(n=15)$ and chronic arthritis $(n=10)$. The figures denote median (range)

\begin{tabular}{|c|c|c|c|c|c|}
\hline & Transient arthritis & & Chronic arthritis & & p value \\
\hline \multirow{5}{*}{ Final diagnosis (number of patients) } & Oligoarthritis & 11 & Oligoarthritis & 3 & \\
\hline & Reactive arthritis & 3 & Rheumatoid arthritis & 3 & \\
\hline & Gout & 1 & Osteoarthritis & 2 & \\
\hline & & & Psoriatic arthritis & 1 & \\
\hline & & & Seronegative large joint arthritis & 1 & \\
\hline Age & $41(25-75)$ & & $50(34-75)$ & & NS \\
\hline Sex (male/female) & $8 / 7$ & & $4 / 6$ & & NS \\
\hline Duration of synovitis (months) & $0.5(0.03-2)$ & & $0.75(0.25-5)$ & & NS \\
\hline Number of swollen joints & $1(1-3)$ & & $2(1-4)$ & & NS \\
\hline $\operatorname{ESR}(\mathrm{mm} / 1$ st hour $)$ & $20(3-27)$ & & $20(5-50)$ & & NS \\
\hline Haemoglobin $(\mathrm{g} / \mathrm{l})$ & $136(106-153)$ & & $134(100-150)$ & & NS \\
\hline SF-aggrecan $(\mu \mathrm{g} / \mathrm{ml})$ & $182.5(78.2-328.8)$ & & $71.3(26.3-166.9)$ & & 0.0007 \\
\hline $\mathrm{SF}-\mathrm{COMP}(\mu \mathrm{g} / \mathrm{ml})$ & $111.7(54.1-226.8)$ & & $98.9(38.3-199.6)$ & & NS \\
\hline $\mathrm{SF}$-aggrecan/COMP concentration ratio & $1.7(0.8-3.9)$ & & $0.8(0.4-1.0)$ & & 0.0001 \\
\hline
\end{tabular}

because of a synovitis of the knee joint during a five year period between 1983-88 was performed. Eighty patients were classified as having knee joint arthritis of unknown cause at the time of joint aspiration. The charts of these patients were surveyed. The requirements for inclusion in the study were determined beforehand. To be included and further studied we required that the chart contained a description of typical signs of synovitis, a duration of symptoms as described by the patient not exceeding six months, and lack of a previous history of arthritis. We permitted presence of symptoms from three additional joints. The patient charts were used to determine the subsequent evolution of the joint disorder. In 13 cases this was not possible because information was only available from the period around the time of presentation at the clinic. These 13 patients were approached by phone and interviewed in 1995 concerning persistent joint symptoms and contacts with other hospitals. RA was diagnosed according to the ACR criteria, ${ }^{9}$ psoriatic arthritis if typical skin symptoms were found in combination with a Waaler-Rose negative arthritis, gout if intracellular urate crystals were identified in synovial fluid obtained from an acutely inflamed joint, and osteoarthritis if radiographic changes compatible with this disorder were present. Reactive arthritis was diagnosed according to Ahvonen et al. ${ }^{10}$

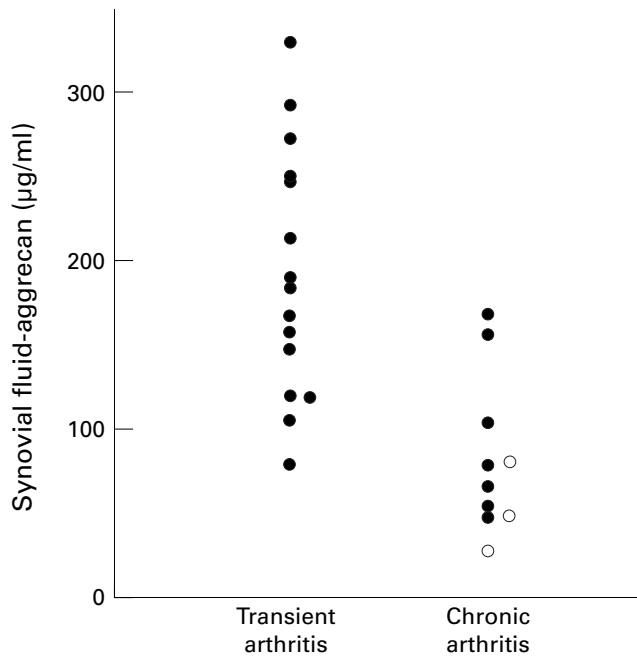

Figure 1 Synovial fluid concentrations of aggrecan in patients with transient or chronic arthritis. The empty circles denote the patients who developed $R A$.
SAMPLE COLLECTION AND IMMUNOASSAYS Synovial fluid samples were obtained, treated, and stored as previously described. ${ }^{11}$

Aggrecan and COMP were quantified by enzyme linked immunosorbent assays using specific polyclonal antisera. ${ }^{11}{ }^{12}$ The aggrecan antiserum recognises epitopes in the core protein of the molecule. Analyses were performed without knowledge of the clinical characteristics of the patients.

STATISTICAL CALCULATIONS

Comparisons between groups were calculated using the Mann-Whitney U test (two tailed) except for sex where the $\chi^{2}$ test was used. A p value of $<0.05$ was considered significant.

\section{Results}

At the initial computer search we found 80 patients who at the time of joint fluid aspiration had been diagnosed as having knee joint arthritis of unknown cause with a duration of synovitis not exceeding six months. By examining their charts it was found that 36 patients already at the time of aspiration had an established diagnosis. In 14 cases there was a history of previous joint symptoms. Three patients had died and were not possible to evaluate. In one patient the arthritis was considered to be result from a loose body and in another case the arthritis was probably a paramalignant reaction. These patients were not studied further.

The remaining 25 patients were further studied and could be separated in two groups according to the final diagnosis as shown in table 1. Thus we were able to identify one group ( $n=15)$ with transient arthritis and one group $(\mathrm{n}=10)$ with chronic joint symptoms.

Table 1 also shows a comparison of some of the features of the patients with transient arthritis and chronic arthritis, respectively, at the time of joint fluid aspiration. No significant difference between the groups were found for the clinical variables, erythrocyte sedimentation rate or haemoglobin concentration. In contrast, the synovial fluid concentrations of aggrecan were significantly higher in the patients with transient arthritis (fig 1). The COMP concentrations did not differ between the groups. To reduce the variability resulting from varying amounts of synovial fluid present in the different joints we also calculated the ratios between the aggrecan and COMP concentrations. The ratios were significantly 


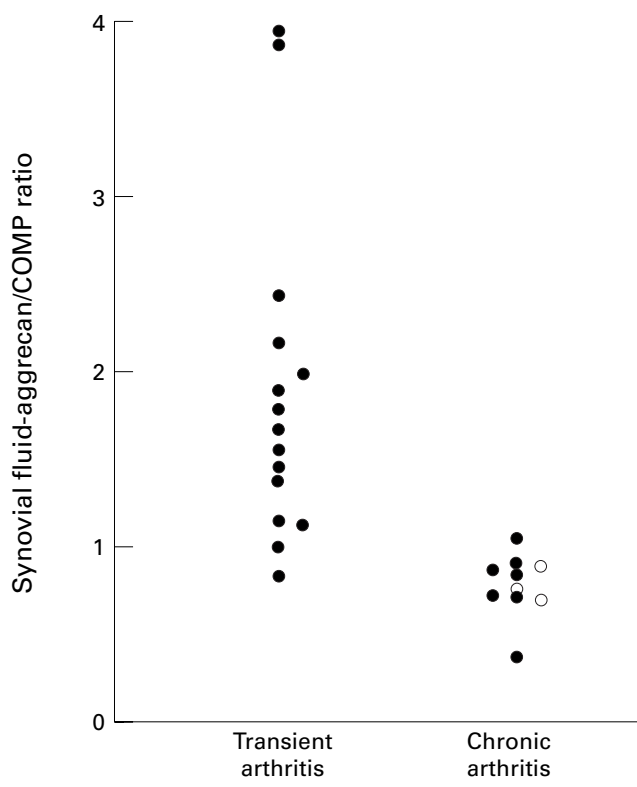

Figure 2 Synovial fluid aggrecan/COMP ratio in patients with transient or chronic arthritis. The empty circles denote the patients who developed $R A$.

higher in the group with transient arthritis, corroborating the different joint fluid pattern of the cartilage markers in the groups (fig 2).

\section{Discussion}

This retrospective follow up of patients who presented with a first time oligoarthritis $7-11$ years earlier indicates that quantification of aggrecan in synovial fluid in the acute stage is a possible way of differentiating between patients with a benign, resolving course and those prone to develop a chronic joint disorder. The use of concentrations for comparisons might be a confounding factor if there were considerable differences in the amount of synovial fluid present in the joints. To circumvent this we also calculated the aggrecan/COMP ratio. This strengthened the findings, indicating a different turnover of aggrecan in the two groups. Thus low concentrations of aggrecan in synovial fluid in acute arthritis could be an early sign of developing chronic arthritis. Notably, three patients in this small study developed RA, two Waaler-Rose positive and one negative. This finding is somewhat in contrast with a previous study, which showed that patients presenting with knee joint arthritis who subsequently developed RA all had symmetrical polyarthritis at presentation. ${ }^{13}$ It should however be noted that all the three patients in our study had oligoarthritis. Symptoms were apparent in the other knee in two patients. One of these also had synovitis in two MCP joints. The third patient had synovitis in a wrist and an ankle in addition to the knee joint.

In view of our previous findings of high aggrecan values in the acute stages of reactive arthritis it may be speculated that most of the
11 patients with transient arthritis in whom no diagnosis was possible were suffering from this condition. ${ }^{67}$ However, no systematic search for aetiological agents was performed because of the retrospective nature of the study. It may be hypothesised that the high synovial fluid content of aggrecan fragments in the transient arthritides reflects release of newly synthesised aggrecan molecules not incorporated in the matrix, which could indicate that reparative processes efficiently prevent further cartilage degradation in these benign cases. (Månsson B, Saxne T, unpublished observations.) On the other hand, in the patients developing chronic conditions, the capacity to repair may be inadequate and the aggrecan fragments released perhaps predominantly reflect degradation of structural molecules. Future studies using antibodies with affinity for fragments of structural molecules or such newly synthesised may verify this hypothesis.

In conclusion, this study has for the first time shown that the synovial fluid content of aggrecan in patients with acute onset arthritis is potentially useful for distinguishing patients with a benign disease course from those who will develop a chronic joint disorder, including RA. This finding, if substantiated in a prospective study, should increase the possibility to initiate early treatment in select cases.

This study was supported by the The Medical Faculty of Lund, The Swedish Medical Research Council, the Österlund, Kock, Nanna Svartz and Anna Greta Crafoord Foundations, the King Gustaf V 80-year Fund and Riksförbundet mot Reumatism. Skilful technical assistance by Mrs Mette Lindell is appreciated.

1 Mau W, Raspe HH, Mersjann H. Early arthritides: nosography, nosology, and diagnostic criteria. Scand J Rheumatol 1989;suppl 79:3-12

2 Kvien TK, Glennås A, Melby K. Prediction of diagnosis in acute and subacute oligoarthritis of unknown origin. $\mathrm{Br} \mathrm{J}$ Rheumatol 1996;35:359-63.

3 Emery P. The optimal management of early rheumatoid disease: the key to preventing disability. $\mathrm{Br} \mathrm{J}$ Rheumatol 1994;33:765-8.

4 Poole AR, Dieppe P. Biological markers in rheumatoid athritis. Semin Arthritis Rheum 1994;23:17-31.

5 Saxne T, Heinegård D. Matrix proteins: potentials as body fluid markers of changes in the metabolism of cartilage and bone in arthritis. J Rheumatol 1995;22 (suppl 43):71-4.

bone in arthritis. J Rheumatol 1995;22 (suppl 43):71-4.
6 Saxne T, Heinegard D, Wollheim FA, Pettersson H. Differences in cartilage proteoglycan level in synovial fluid in early rheumatoid arthritis and reactive arthritis. Lancet 1985; ;ii: $127-8$

7 Saxne T, Glennås A, Kvien TK, Melby K, Heinegård D. Release of cartilage macromolecules into synovial fluid in acute and prolonged stages of reactive arthritis. Arthritis Rheum 1993;36:20-5.

8 Månsson $\mathrm{B}$, Geborek $\mathrm{P}$, Saxne $\mathrm{T}$. Cartilage and bone macromolecules in knee joint synovial fluid in rheumatoid arthritis: relation to development of knee or hip joint destruction. Ann Rheum Dis 1997;56:91-6.

9 Arnett FC, Edworthy SM, Bloch DA, McShane DJ, Fries $\mathrm{JF}$, Cooper NS, et al. The American Rheumatism Association 1987 revised criteria for classification of rheumatoid arthritis. Arthritis Rheum 1988;31:315-24.

10 Ahvonen P, Sievers K, Aho K. Arthritis associated with Yersinia entercolitica infections. Acta Rheumatol Scand 1969;15:232-55.

11 Saxne T, Heinegård D, Wollheim FA. Therapeutic effects on cartilage metabolism in arthritis as measured by release of proteoglycan structures into the synovial fluid. Ann Rheum Dis 1986;45:491-7.

12 Saxne T, Heinegård D. Cartilage oligomeric matrix protein: a novel marker of cartilage detectable in synovial fluid and blood. Br J Rheumatol 1992;31:583-91.

13 Devlin J, Gough A, Huisson A, Jubb R, Emery P. Knee disease in 766 early arthritis patients, relevance to outcome and pathogenesis. Arthritis Rheum 1993;36 (suppl 9):262. 